\title{
Knockdown of Rhotekin 2 expression suppresses proliferation and invasion and induces apoptosis in hepatocellular carcinoma cells
}

\author{
WEI WEI ${ }^{1,2^{*}}$, HUABING CHEN ${ }^{1,2^{*}}$ and SIBIN LIU ${ }^{1,2}$ \\ ${ }^{1}$ Department of Radiology, Jingzhou Central Hospital; ${ }^{2}$ Tongji Medical College, \\ Huazhong University of Science and Technology, Jingzhou, Hubei 434020, P.R. China
}

Received February 10, 2015; Accepted December 3, 2015

DOI: $10.3892 / \mathrm{mmr} .2016 .5113$

\begin{abstract}
Hepatocellular carcinoma (HCC), which is one of the most common types of cancer worldwide, has been ranked as the third leading cause of cancer-associated mortality worldwide. Rhotekin 2 (RTKN2), a Rho-guanosine triphosphatase (GTPase) effector, has been reported to be anti-apoptotic. However, the molecular mechanism underlying the biological function of RTKN2 in HCC is poorly defined. The current study reported that RTKN2 was overexpressed in $83 \%$ of HCC specimens compared with adjacent noncancerous tissues $(n=30)$. Depletion of RTKN2 in HCC cells, HepG2 and BEL-7404 by RNA interference led to marked inhibition of cell proliferation and cell cycle progression. Notably, RTKN2 silencing significantly reduced the levels of cell cycle-associated proteins, proliferating cell nuclear antigen and cyclin-dependent kinase 1 . Additionally, it was identified that downregulation of RTKN2 in HCC cells notably induced cell apoptosis, while significantly repressing cell invasion. These data suggest that RTKN2 may act as an oncogene and inhibition of RTKN2 may be part of a novel therapeutic strategy for targeted HCC therapy.
\end{abstract}

\section{Introduction}

Hepatocellular carcinoma (HCC), which accounts for between 85 and $90 \%$ of cases of primary liver cancer, is the fifth most common cancer worldwide and is currently the third leading cause of cancer-associated mortality (1). The prognosis of patients with HCC continues to be poor, despite improving treatment approaches, with a 5-year overall survival less than $30 \%$ (2). The curative rate of early-stage HCC has been improved by the use of liver resection or transplantation.

Correspondence to: Dr Sibin Liu, Department of Radiology, Jingzhou Central Hospital, 1 Renmin Road, Jingzhou, Hubei 434020, P.R. China

E-mail: liusibin188@163.com

${ }^{*}$ Contributed equally

Key words: Rhotekin 2, hepatocellular carcinoma, proliferation, apoptosis, invasion
However, the curative efficacy of these therapies is limited due to the fact that the majority of patients with HCC are diagnosed at a late stage with severe intrahepatic and extrahepatic metastases (3). Novel diagnostic markers, prognostic indicators and effective therapeutic targets are required, thus it is important to investigate the underlying mechanisms of the pathogenesis of HCC.

Rhotekin 2 (RTKN2) is a member of the rhotekin protein family. The two rhotekin proteins, RTKN and RTKN2, have an N-terminal HR1 domain (Rho-GTPase binding domain) and pleckstrin homology domain (4). The similar protein architecture of rhotekin proteins indicated that they may share functional characteristics (4). The HR1 domain of murine RTKN has been demonstrated to bind to Ras homolog gene family member A (Rho A) and to inhibit the GTPase activity of Rho A (5). Rho proteins are crucial in cell cytoskeletal organization, cell growth, cell differentiation and transformation. Dysregulation of the Rho signal transduction pathway has been implicated in numerous types of cancer (6). The inhibitory role of RTKN2 on Rho A has previously indicated an important function for rhotekin proteins in cancer (5).

Previous studies have demonstrated an anti-apoptotic role for rhotekin $(7,8)$. Liu et al $(8)$ reported the overexpression of RTKN in the majority of gastric cancer patients and demonstrated an association between RTKN expression and metastatic progression. Stable RTKN-expressing gastric cells have been reported to be resistant to apoptosis induced by sodium butyrate and serum deprivation (7). A previous study reported that overexpression of RTKN resulted in the activation of nuclear factor (NF)- $\mathrm{B}$ and an induction of a number of NF- $\kappa \mathrm{B}$-regulated anti-apoptotic genes (7). Conversely, suppressing expression of RTKN by short interfering RNA (siRNA) greatly sensitized cells to apoptosis (7). Similarly, several studies have been conducted concerning the anti-apoptotic effects of RTKN2 (9-12). RTKN2 was originally identified in a promyelocytic cell line resistant to 25-OHC-induced apoptosis (12). RTKN2 has been reported to serve a role in the process of intrinsic apoptosis of HEK cells via activating $\mathrm{NF}-\kappa \mathrm{B}$ signaling (10). On the contrary, suppression of RTKN2 in primary human CD4+ lymphocytes reduced viability and increased sensitivity to 25-OHC (10). Collectively, the previous studies demonstrated the anti-apoptotic role of RTKN2. However, little is known about the expression pattern and the role of RTKN2 in cancer, particularly in HCC. 
In the present study, the role of RTKN2 in HCC and the associated mechanisms were investigated. Firstly, we found that the mRNA level of RTKN2 was significantly increased in HCC tissues compared to non-tumorous tissues. Then we investigated the role of RTKN2 in multiple cellular progress including cell proliferation, cell cycle, apoptosis and invasion.

\section{Materials and methods}

Patients and tissue samples. Tumor tissues and paired noncancerous tissues were collected from 30 patients with HCC admitted to the Department of Radiology, Jingzhou Central Hospital (Jingzhou, China) between 2010 and 2012. Ethical approval for the study was provided by the Independent Ethics Committee of Jingzhou Central Hospital. Informed and written consent was obtained from all patients or their advisers according to the ethics committee guidelines.

Cell lines. HEK293T, HCC, HepG2, HuH7, BEL-7404, SMMC-7721, MHCC-97L and MHCC-97H cells were obtained from the Cell Bank of Shanghai Biology Institute, Chinese Academy of Science (Shanghai, China). All culture media were supplemented with $10 \%$ fetal bovine serum, $100 \mathrm{mg} / \mathrm{ml}$ penicillin $\mathrm{G}$ and $50 \mu \mathrm{g} / \mathrm{ml}$ streptomycin (Life Technologies; Thermo Fisher Scientific, Inc., Waltham, MA, USA). HEK293T, HepG2, HuH7, BEL-7404, MHCC-97L and MHCC-97H cells were cultured in Dulbecco's modified Eagle's medium (DMEM; Life Technologies; Thermo Fisher Scientific, Inc.) and the SMMC-7721 cells were cultured in Roswell Park Memorial Institute 1640 medium (Life Technologies; Thermo Fisher Scientific, Inc.). All cells were maintained at $37^{\circ} \mathrm{C}$ in $5 \% \mathrm{CO}_{2}$.

Vector construction. pLKO.1, psPAX2 and pMD2.G were purchased from Addgene, Inc. (Cambridge, MA, USA). Three small hairpin RNAs (shRNAs; Generay Biotech Co., Ltd., Shanghai, China) targeting human RTKN2 mRNA were cloned into a lentiviral vector (PLKO.1). A non-specific scramble shRNA sequence (CCTAAGGTTAAGTCGCCCTCG) was used as negative control. The constructs were then transfected into HEK293T cells with lentiviral packaging vectors (psPAX2 and pMD2.G) using Lipofectamine 2000 (Invitrogen; Thermo Fisher Scientific, Inc.) according to the manufacturer's instructions. Viruses were collected $48 \mathrm{~h}$ subsequent to transfection and used to infect HepG2 and BEL-7404 cells. After 48 h, the cells were processed for reverse transcription-quantitative polymerase chain reaction (RT-qPCR) and western blotting. The shRNA target sequences for RTKN2 were listed in Table I.

RNA extraction and RT-qPCR. Total RNA was isolated using TRIzol reagent (Invitrogen; Thermo Fisher Scientific, Inc.) according to the manufacturer's instructions. cDNA was synthesized using $1 \mu \mathrm{g}$ total RNA, $0.5 \mathrm{mM}$ dNTPs, $2.5 \mu \mathrm{M}$ oligo-dT primer (for mature mRNAs), $1 \mathrm{U} / \mu 1$ RiboLock RNase Inhibitor and $10 \mathrm{U} / \mu \mathrm{l}$ M-MuLV Reverse Transcriptase (all from Thermo Fisher Scientific, Inc.). The cDNA synthesis was performed for $1 \mathrm{~h}$ at $37^{\circ} \mathrm{C}$ and the reaction was stopped by 10 min incubation at $70^{\circ} \mathrm{C}$.

RT-qPCR was performed in an Applied Biosystems 7300 Fast Real-Time PCR System (Applied Biosystems; Thermo
Fisher Scientific, Inc.) using the Absolute Blue qPCR SYBR Green Low ROX mix (Thermo Fisher Scientific, Inc.). For each reaction $5 \mu \mathrm{l}$ cDNA template, $1 \mu \mathrm{l}$ primer pairs $(10 \mu \mathrm{M})$ and $10 \mu \mathrm{l}$ of the qPCR SYBR mixture to a final reaction volume of $20 \mu \mathrm{l}$ were used. All reactions were conducted using the following cycling parameters: $95^{\circ} \mathrm{C}$ for $10 \mathrm{~min}$, followed by 40 cycles of $95^{\circ} \mathrm{C}$ for $15 \mathrm{sec}$ and $60^{\circ} \mathrm{C}$ for $45 \mathrm{sec}$, and a final extension step at $72^{\circ} \mathrm{C}$ for $5 \mathrm{~min}$. Relative expression levels at the tested experimental conditions were calculated within each independent experiment using the using the $\Delta \Delta \mathrm{Cq}$ method (13). All data represent the average of three replicates. The quality of the PCR product was monitored using post-PCR melt curve analysis. The sequences of the primer pairs used are as follows: RTKN2 (NM_001282941.1), F 5'-ACAGTT CGCGTTGGAGATGGAG-3' and R 5'-GTCGAGCATTGC ACACCATGAG-3'; glyceraldehyde 3-phosphate dehydrogenase (GAPDH) (NM_001256799.1), F 5'-CACCCACTCCTC CACCTTTG-3' and R 5'-CCACCACCCTGTTGCTGTAG-3'.

Western blotting. Treated and untreated HepG2 and BEL-7404 cells were washed twice with phosphate-buffered saline (PBS) and lysed in ice-cold radioimmunoprecipitation assay buffer (JRDUN Biotechnology Co., Ltd., Shanghai, China) with freshly added $0.01 \%$ protease inhibitor cocktail (Sigma-Aldrich, St. Louis, MO, USA) and incubated on ice for $30 \mathrm{~min}$. Subsequent to centrifugation, supernatants were collected and stored at $-80^{\circ} \mathrm{C}$ until required for use. For western blot analysis, the cell lysates were run on a $10-15 \%$ sodium dodecyl sulfate-polyacrylimide gel electrophoresis gel and transferred electrophoretically to a nitrocellulose membrane (EMD Millipore, Billerica, MA, USA). The blots were blocked with $5 \%$ skimmed milk with gentle shaking, followed by incubation with primary antibodies. Blots were then incubated with the horseradish peroxidase-conjugated goat anti-mouse (cat. no. A0216; dilution 1:1,000) or goat anti-rabbit (cat. no. A0208; dilution 1:1,000) secondary antibodies (Beyotime Institute of Biotechnology, Shanghai, China) then were visualized using enhanced chemiluminescence (ECL; EMD Millipore). Mouse monoclonal anti-PTKN2 (cat. no. ab118069; dilution 1:200), rabbit monoclonal anti-proliferating cell nuclear antigen (PCNA; cat. no. ab92552; 1:5,000) and mouse monoclonal anti-cyclin-dependent kinase 1 (CDK1; cat. no. ab18; 1:1,000) antibodies were purchased from Abcam (Cambridge, MA, USA). The rabbit monoclonal anti-GAPDH antibody (cat. no. 5174; 1:1,500) was from Cell Signaling Technology, Inc. (Danvers, MA, USA).

Determination of cell proliferation. Cell proliferation was measured using the Cell-Counting Kit-8 (CCK-8) Assay kit (Dojindo Molecular Technologies, Kumamoto, Japan) according to the manufacturer's instructions. In brief, the treated and untreated HepG2 and BEL-7404 cells were seeded onto 96-well plates. At the indicated time points, CCK-8 solution (10 $\mu \mathrm{l}$ in $100 \mu \mathrm{l}$ DMEM) was added to each well and incubated for $1 \mathrm{~h}$. Optical density values at a wavelength $450 \mathrm{~nm}$ were measured using a microplate reader (model 550; Bio-Rad Laboratories, Inc., Hercules, CA, USA). All experiments were run in triplicate and repeated a minimum of three times. 
Table I. shRNA target sequences for RTKN2.

\begin{tabular}{lc}
\hline $\begin{array}{l}\text { shRNA } \\
\text { (targeting position) }\end{array}$ & \multicolumn{1}{c}{ Sequence } \\
\hline $\begin{array}{l}\text { RTKN2-RNAi-1 } \\
(385-407)\end{array}$ & GAGCACTCAGAAAGATCAAGTCT \\
RTKN2-RNAi-2 & AAAGGTTCTATGTGCCTAAATCT \\
$(1418-1440)$ & \\
RTKN2-RNAi-3 & CAGGGAAAGAACAATAG AAGTCT \\
$(1967-1989)$ & \\
\hline
\end{tabular}

shRNA, small hairpin RNA; RTKN2, Rhotekin 2.

Cell cycle distribution assay. The cell cycle was analyzed as previously described (14) with slight modifications. Briefly, cells cultured on $60-\mathrm{mm}$ diameter dish were infected with the indicated virus. After $48 \mathrm{~h}$, cells were harvested by trypsinization (Beijing Solarbio Science \& Technology Co., Ltd., Beijing, China), washed with ice-cold PBS, and fixed with ice-cold $70 \%$ ethanol for a minimum of $2 \mathrm{~h}$ at $-20^{\circ} \mathrm{C}$. The fixed cells were washed with $\mathrm{PBS}$, incubated with ribonuclease A (Sigma-Aldrich) and propidium iodide (PI; $0.05 \mathrm{mg} / \mathrm{ml}$, Sigma-Aldrich) at room temperature in the dark for $30 \mathrm{~min}$. DNA content was then analyzed using a FACScan flow cytometer (BD Biosciences, San Jose, CA, USA). The percentage of cells in the $G_{0} / G_{1}, S$ and $G_{2} / M$ phases were determined with FlowJo software version 7.6 (Tree Star, Inc., Ashland, OR, USA). Experiments were performed in triplicate and $3 \times 10^{4}$ cells were analyzed per sample.

Cell apoptosis assay. The percentage of cells actively undergoing apoptosis was determined by double staining with annexin V-fluorescein isothiocyanate (FITC; BD Biosciences) and PI (Sigma-Aldrich). Cells were cultured on $60-\mathrm{mm}$ diameter dishes and were infected with the indicated viruses. After 48 h, cells were harvested and then double-labeled with annexin V-FITC and PI as described by the manufacturer. Cells were analyzed using a FACScan flow cytometer.

Boyden chamber invasion assay. Invasion chambers (BD Biosciences) were prepared by coating the membranes ( $8 \mathrm{~mm}$ pores) of 24 -well inserts with $10 \mathrm{mg} / \mathrm{ml}$ Matrigel (BD Biosciences) leaving them to set for $1 \mathrm{~h}$ at $37^{\circ} \mathrm{C}$. Indicated cells were serum starved for $24 \mathrm{~h}$, then a total of $5 \times 10^{4}$ cells were added to the upper chamber and suspended in $200 \mu \mathrm{l}$ DMEM, with $500 \mu \mathrm{l}$ serum-free DMEM added into the lower chamber. Subsequent to $48 \mathrm{~h}$ of incubation, the cells on the upper surface of the filter were completely removed using a cotton bud. The remaining invaded cells were washed with PBS, fixed in 4\% paraformaldehyde (Beijing Solarbio Science \& Technology Co., Ltd.) and then were stained with $0.2 \%$ crystal violet (Beijing Solarbio Science \& Technology Co., Ltd.). The invading cells were observed under the microscope (ECLIPSE Ti-S; Nikon Corporation, Tokyo, Japan) at a magnification of $\mathrm{x} 200$ in 10 different fields from each filter. Cells were counted in the central field of triplicate membranes. Experiments were repeated three times.
A

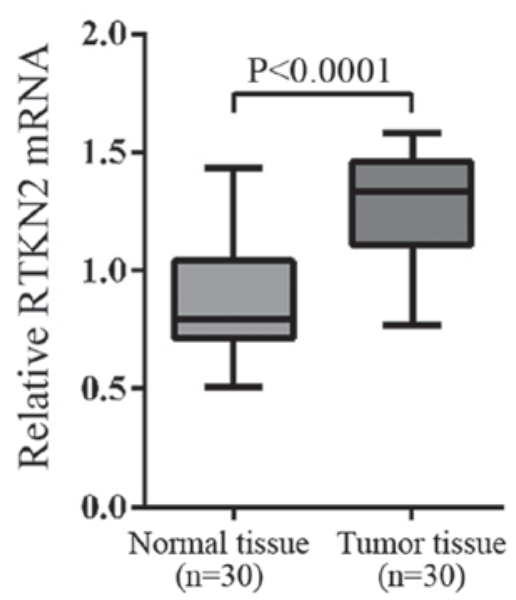

$\mathbf{B}$

\section{Data from TCGA HCC dataset}

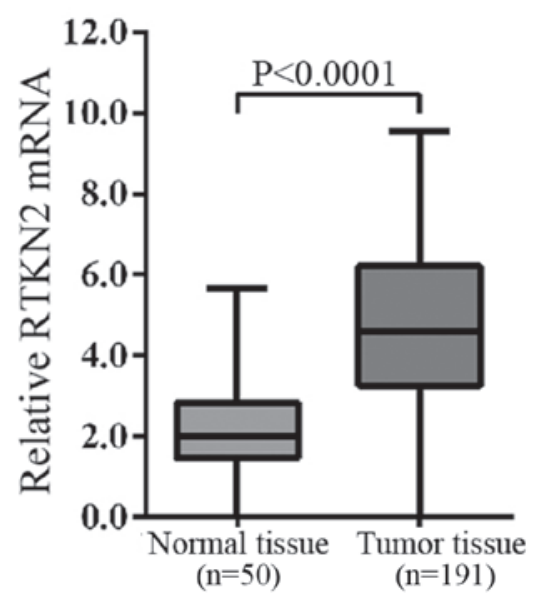

Figure 1. RTKN2 was overexpressed in HCC tissues. (A) The expression level of RTKN2 was determined by reverse transcription-quantitative polymerase chain reaction in 30 pairs of HCC and noncancerous tissues collected from patients admitted to Jinzhou Central Hospital between 2010 and 2012 $(\mathrm{P}<0.0001)$. (B) RTKN2 expression was significantly increased in HCC tumor tissues when compared with the adjacent tissues of patients from the TCGA HCC cohort $(\mathrm{P}<0.0001)$. RTKN2, Rhotekin 2; HCC, hepatocellular carcinoma; TCGA, The Cancer Genomic Atlas.

Statistical analysis. All data are presented as the mean \pm standard deviation. Statistical significance was determined by Student's two-tailed t-test. $\mathrm{P}<0.05$ was considered to indicate a statistically significant difference.

\section{Results}

Upregulated RTKN2 expression was observed in HCC. The mRNA expression levels of RTKN2 were compared between HCC tissues and the adjacent normal tissues using RT-qPCR. Overexpression of RTKN2 was identified in $83 \%(25 / 30)$ of the measured HCC tissues. Statistical analysis using Student's t-test demonstrated that the RTKN2 mRNA expression levels were significantly overexpressed in HCC tissues when compared with normal tissues (Fig. 1A; P<0.0001).

In addition, the RNA-sequencing data of the HCC cohort of The Cancer Genome Atlas project (15) was analyzed and it 
A
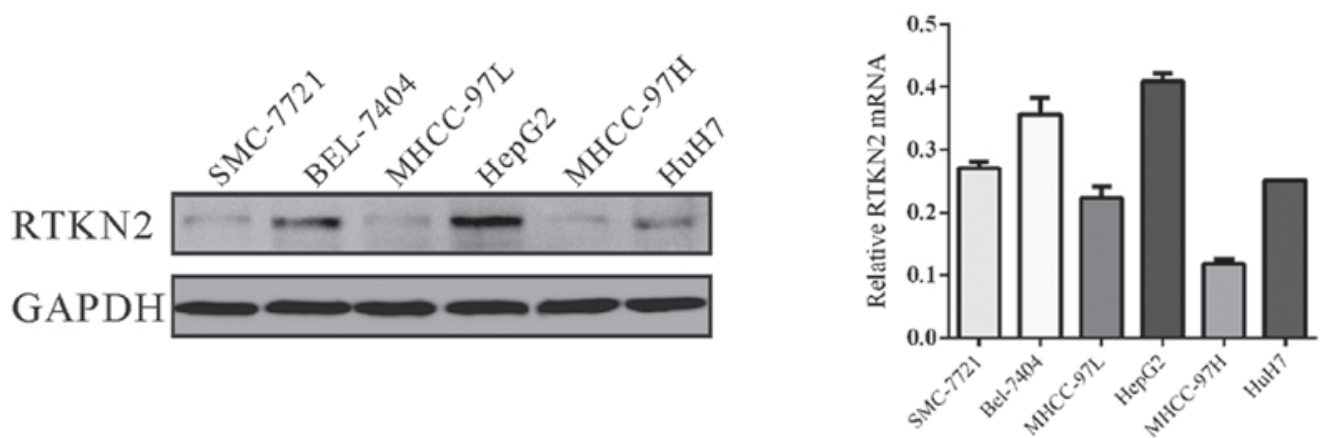

B

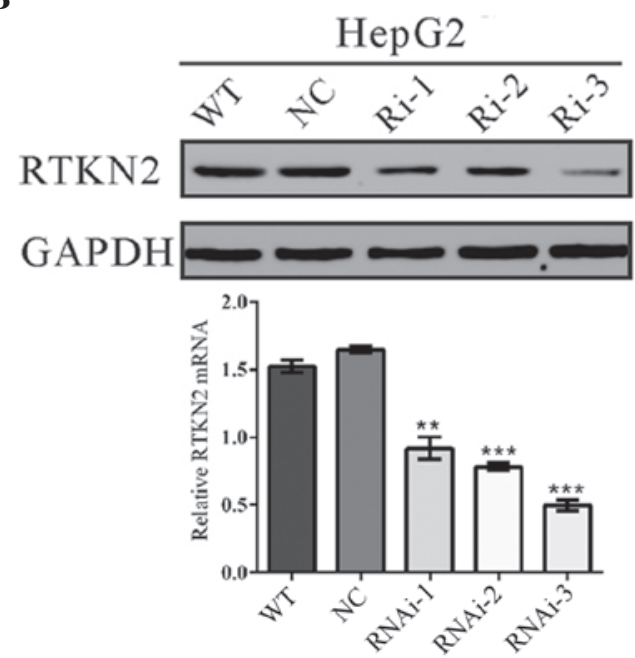

C

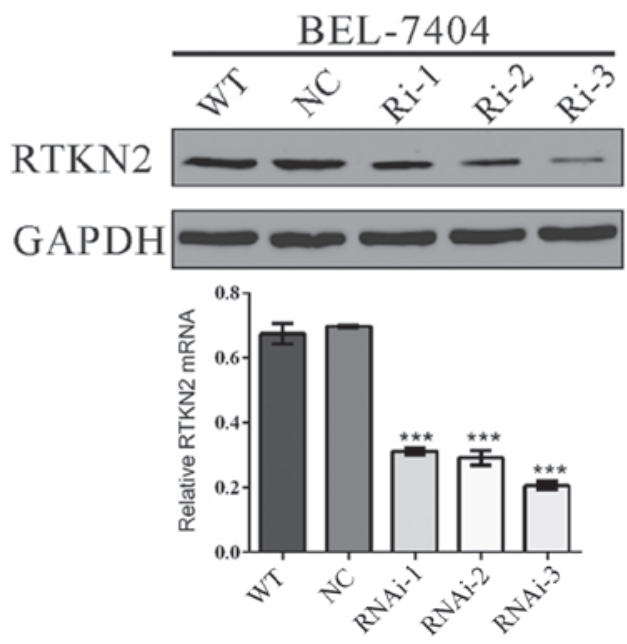

Figure 2. RTKN2 was suppressed by RNAi in HCC cells. (A) RTKN2 expression level in six HCC cell lines was analyzed by western blotting (left panel) and RT-qPCR (right panel). Data were based on a minimum of three independent experiments. Expression of RTKN2 in (B) HepG2 and (C) BEL-7404 cells was analyzed by western blotting (upper panel) and RT-qPCR (lower panel). ${ }^{* *} \mathrm{P}<0.01$ and ${ }^{* * *} \mathrm{P}<0.001$, vs. the NC. RTKN2, Rhotekin 2 ; HCC, hepatocellular carcinoma; RT-qPCR, reverse transcription-quantitative polymerase chain reaction; GAPDH, glyceraldehyde 3-phosphate dehydrogenase; WT, wild type cells; NC, scrambled shRNA virus-infected cells; Ri-1, Ri-2 and Ri-3, RTKN2-shRNA-1, -2 and -3 virus-infected cells.

was identified that RTKN2 expression significantly increased in HCC tumor tissues compared with the adjacent tissues of patients (Fig. 1B).

RTKN2 was downregulated by RNA interference (RNAi) in $H C C$ cells. The expression levels of RTKN2 were evaluated using RT-qPCR and western blotting in the following six HCC cell lines: HepG2, HuH7, BEL-7404, SMMC-7721, MHCC-97L and MHCC-97H. Two cell lines, HepG2 and BEL-7404, exhibited higher RTKN2 mRNA and protein expression, while the remaining four cell lines, HuH7, SMMC-7721, MHCC-97 L and $\mathrm{MHCC}-97 \mathrm{H}$, exhibited reduced mRNA and protein expression (Fig. 2A).

To investigate the functions of RTKN2 on HCC, the HepG2 and MHCC-97H cell lines were selected for further study due to the observed overexpression of RTKN2. Three shRNAs targeting RTKN2 were all able to efficiently suppress endogenous RTKN2 in HepG2 (Fig. 2B) and BEL-7404 cells (Fig. 2C). The RTKN2-Ri-3 shRNA virus was identified to be the most efficient shRNA, thus was stably infected into HepG2 and BEL-7404 cells for further functional analysis.

Suppressing RTKN2 expression inhibited cell proliferation. To examine the effects of RTKN2 downregulation on the proliferation of HCC cells, the proliferation of RTKN2 knockdown cells was assessed using the CCK- 8 assay. As presented in Fig. 3A and B, cell growth was markedly impaired in RTKN2-RNAi virus-infected cells compared with wild type cells (WT) and scramble shRNA virus-infected cells (NC). The data indicated a role for RTKN2 in the promotion of HCC cell proliferation.

Knockdown of RTKN2 repressed $G_{I} / S$ cell cycle transition. The possible effect of RTKN2 knockdown on cell cycle progression was then investigated. PI staining and flow cytometry analysis (Fig. 3C) indicated that the population of RTKN2-RNAi HepG2 cells in $\mathrm{G}_{0} / \mathrm{G}_{1}$ phase was significantly increased by $39.6 \%(\mathrm{P}<0.05)$, and the population of $\mathrm{S}$ phase cells was markedly reduced, by $68.0 \%$, compared with cells infected with scramble shRNA virus-infected cells (NC). Similar results were obtained in BEL-7404 cells (Fig. 3D). These results suggested that RTKN2 promoted $\mathrm{G}_{1} / \mathrm{S}$ cell cycle transition in HCC cells.

The expression levels of cell cycle-associated proteins, PCNA and CDK1 were evaluated by western blotting (Fig. 3E and F). Suppression of RTKN2 expression significantly reduced PCNA expression (16), a marker of cell proliferation, by 53.0 and $69.6 \%$ in HepG2 and BEL-7404 cells, respectively, compared with corresponding NC cells. The protein level of CDK1, which induces mitosis during 
A HepG2

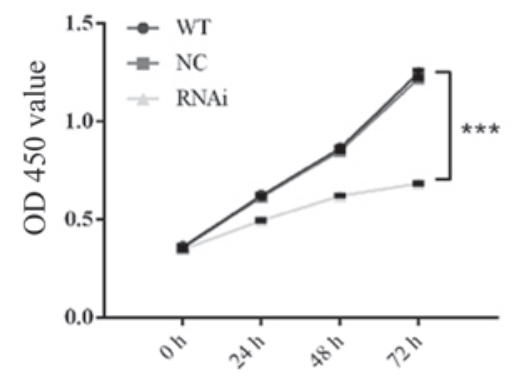

C HepG2

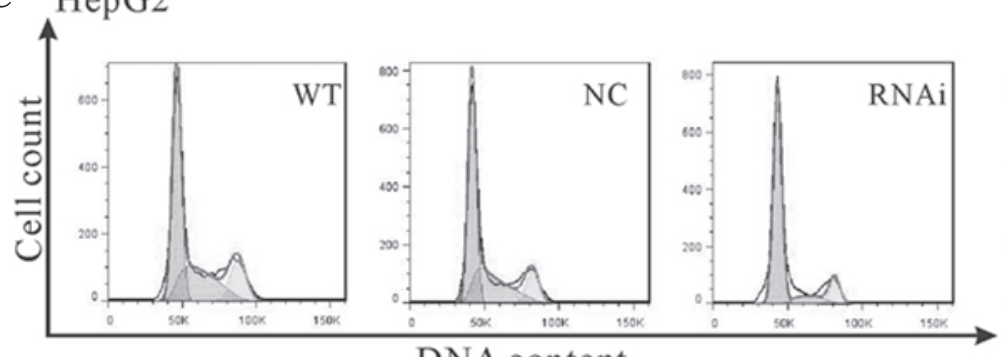

DNA content

D BEL-7404

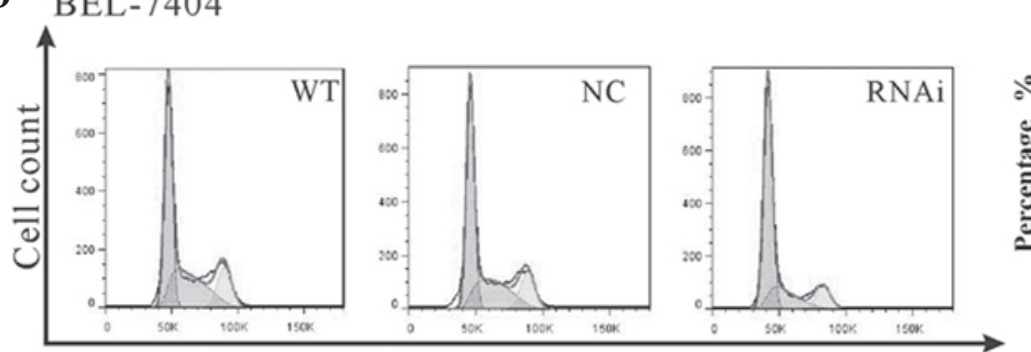

DNA content

$\mathbf{E}$

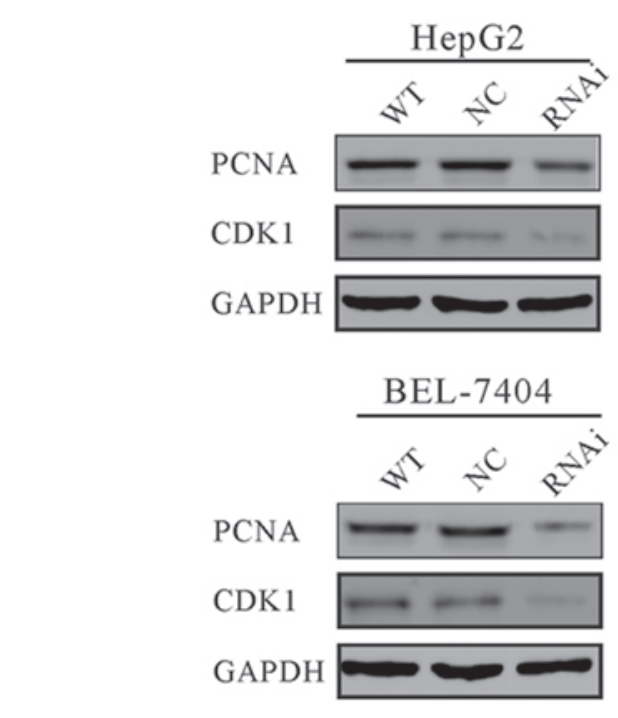

$\mathbf{F}$

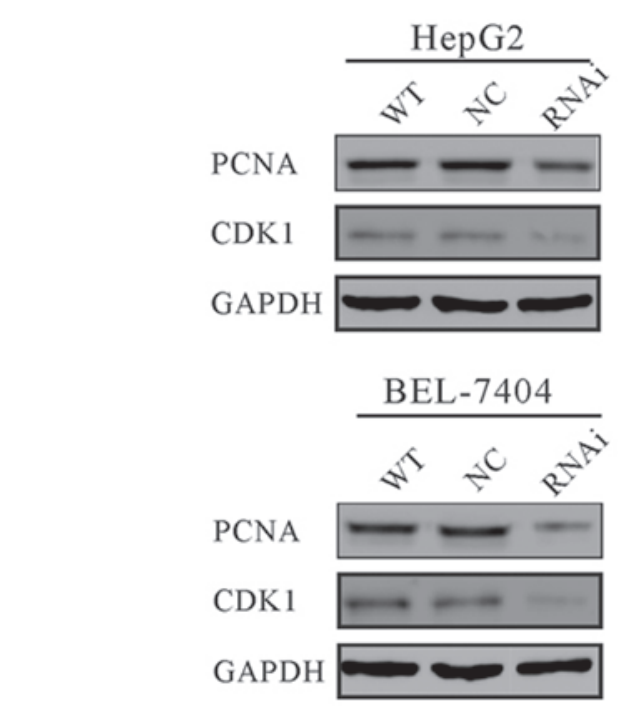

$\mathbf{E}$
B

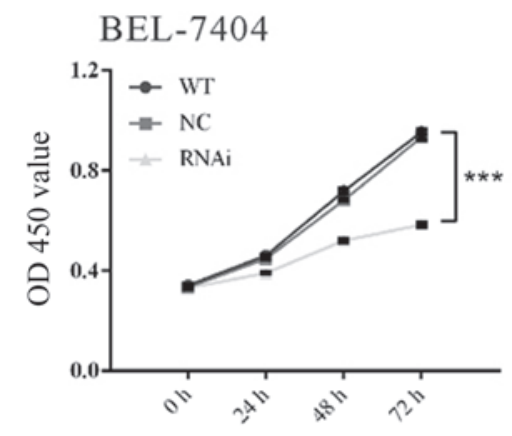

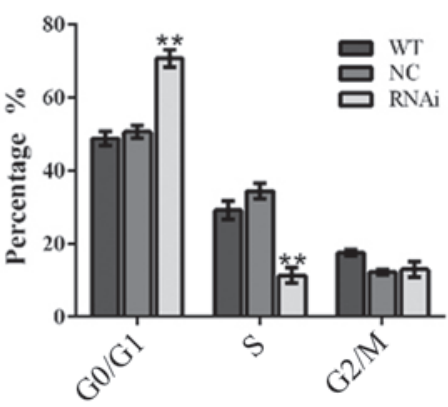

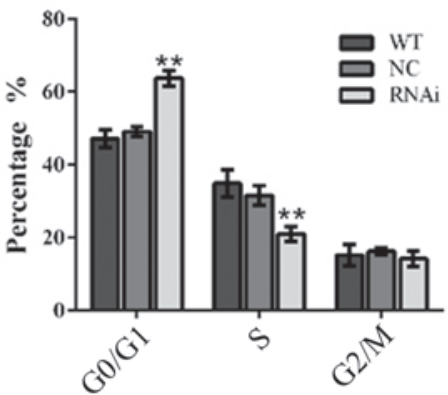

Figure 3. RTKN2 promote cell proliferation by accelerating $\mathrm{G}_{1} / \mathrm{S}$ phase transition in $\mathrm{HCC}$ cells. (A and B) Cell proliferation was detected at $0,24,48$ and $72 \mathrm{~h}$ in HepG2 and BEL-7404 cells. (C and D) The cell cycle profile was analyzed using flow cytometry; (E and F) expression of cell cycle-associated proteins, PCNA and CD1 were evaluated by western blotting. Data were based on a minimum of 3 independent experiments, and presented as the mean \pm standard deviation $\left(\right.$ (*"P $\left.<0.01,{ }^{* * *} \mathrm{P}<0.001\right)$. RTKN2, Rhotekin 2; HCC, hepatocellular carcinoma; OD, optical density; WT, wild type cells; NC, scrambled shRNA virus-infected cells; RNAi, RTKN2-shRNA virus-infected cells.

cell cycle progression (17), was also reduced in RTKN2 knockdown cells. These results suggested that suppression of RTKN2 expression inhibited cell cycle transition in HCC cells.
RTKN2 knockdown induced cell apoptosis. To substantiate the role of RTKN2 on cell apoptosis, annexin V/PI staining was performed (Fig. 4). The ratio of HepG2 cells undergoing apoptosis was significantly increased to $29.4 \pm 0.66 \%$ in 
A

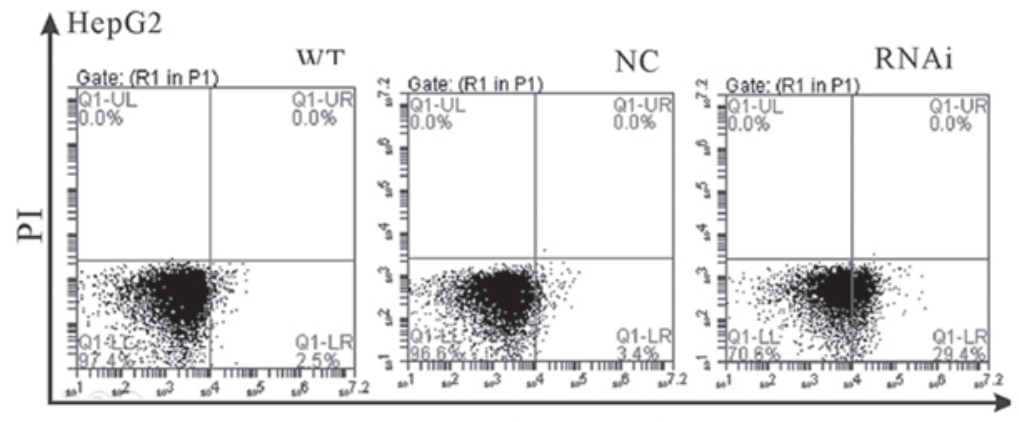

Annexin V-FITC

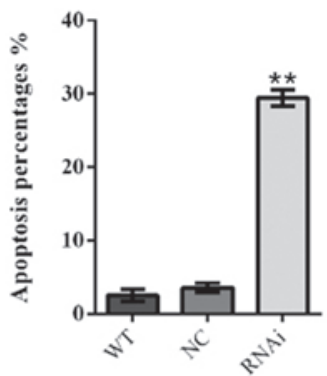

B

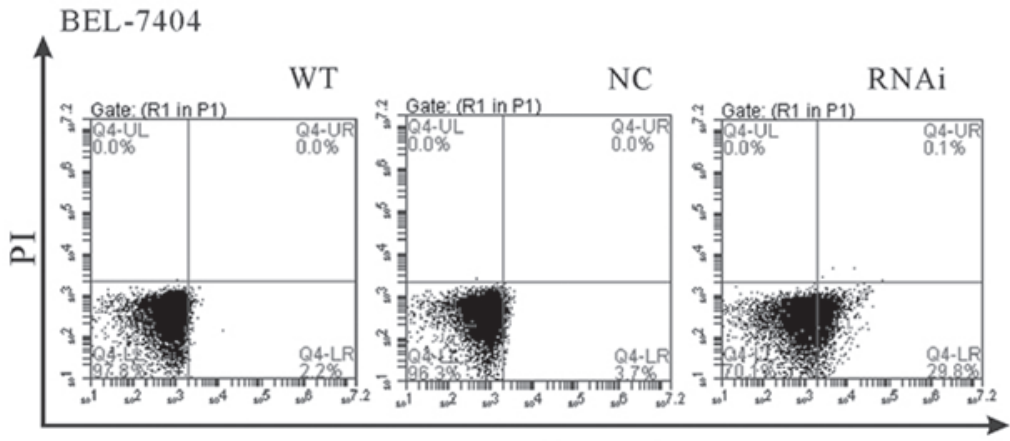

Annexin V-FITC

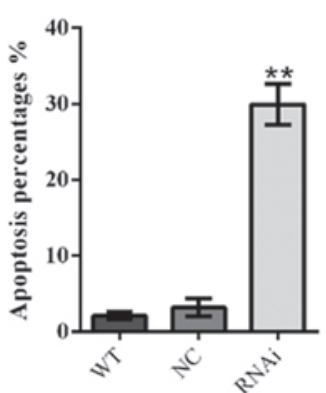

Figure 4. RTKN2 knockdown induced cell apoptosis in HCC cells. (A) HepG2 and (B) BEL-7404 cells were infected with the indicated virus and $48 \mathrm{~h}$ later cells were collected. Cells were stained with annexin V-FITC/PI and apoptosis rates was analyzed using flow cytometry. Data were based on a minimum of 3 independent experiments, and presented as the mean \pm standard deviation $\left({ }^{* *} \mathrm{P}<0.01\right)$. RTKN2, Rhotekin 2 ; HCC, hepatocellular carcinoma; FITC, fluorescein isothiocyanate; PI, propidium iodide; WT, wild type cells; NC, scrambled shRNA virus-infected cells; RNAi, RTKN2-shRNA virus-infected cells.

A HepG2

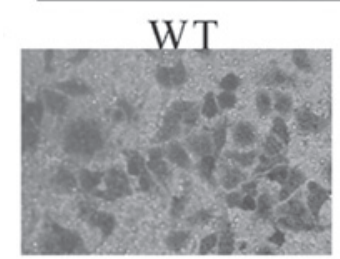

B

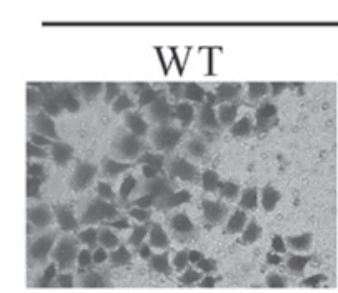

C

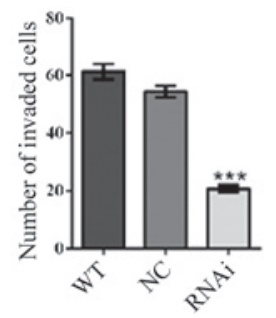

$\mathrm{NC}$

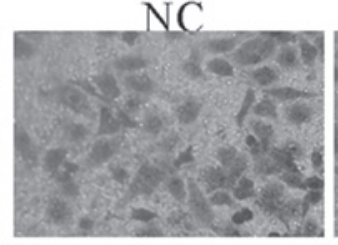

BEL-7404

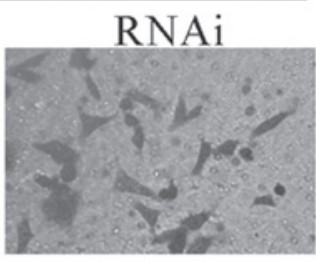

RNAi

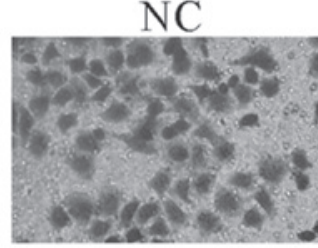

D

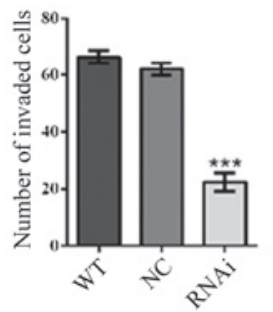

Figure 5. Suppressing RTKN2 expression inhibited cell invasion in HCC cells. Invasion assay of control and RTKN2 knockdown cells in Matrigel-coated transwell chambers. Cells that migrated from the upper well of a transwell chamber into the lower well were stained, photographed and counted. Representative images of cell invasion assay are presented for (A) HepG2 and (B) BEL-7404 cells. Quantitative results of the cell invasion assay for (C) HepG2 and (D) BEL-7404 cells. Data were based on a minimum of 3 independent experiments, and presented as the mean \pm standard deviation $\left({ }^{* * *} \mathrm{P}<0.001\right)$. RTKN2, Rhotekin 2; HCC, hepatocellular carcinoma; WT, wild type cells; NC, scrambled shRNA virus-infected cells; RNAi, RTKN2-shRNA virus-infected cells. 
RTKN2-RNAi cells, compared with WT $(2.5 \pm 0.46 \%)$ and NC cells $(3.53 \pm 0.35 \%)$ (Fig. 4A). Similar results were observed in BEL-7404 cells (Fig. 4B). These data suggested RTKN2 may serve an anti-apoptotic role in HCC cells.

Downregulation of RTKN2 by siRNA inhibited the invasion of $H C C$ cells. To investigate whether RTKN2 affected the invasive ability of HCC cells, a Matrigel-coated membrane chamber invasion assay was conducted (Fig. 5). By contrast to control cells (WT and NC), a significant reduction in the invasive ability was observed in RTKN2 knockdown cells. The number of invaded RTKN2-RNAi cells was less than $40 \%$ of that of NC cells (HepG2 cells: WT, $61 \pm 3$; NC, 54 \pm 2 ; RTKN2-RNAi, $21 \pm 1$. BEL-7404 cells: WT, $66 \pm 2$; NC, 62 \pm 2 ; RTKN2-RNAi, 22 \pm 3 ). These data suggested that RTKN2 promoted $\mathrm{HCC}$ cell invasion.

\section{Discussion}

RTKN2 is a newly identified Rho-GTPase effector protein. Deregulation of Rho GTPase pathways has been reported to serve an important role in tumorigenesis and cancer metastasis of HCC cells $(18,19)$. However, the functional implication of RTKN2 in HCC remains to be fully defined. In the present study, it was identified that RTKN2 was overexpressed in HCC tissues (Fig. 1). The effects of RTKN2 on proliferation, cell cycle, apoptosis and invasion were then investigated by suppressing its expression in HepG2 and BEL-7404 cells, which have higher levels of endogenous RTKN2 expression. It was observed that knockdown of RTKN2 significantly reduced cell growth (Fig. 3A and B). Furthermore, cell cycle analysis (Fig. 3C and D) indicated that suppression of RTKN2 expression in HCC cells inhibited cell cycle progression. The expression of the cell cycle regulators, CDK1 and PCNA, was additionally demonstrated (Fig. 3E and F). CDK1 forms a heterodimer with cyclin $\mathrm{B}$, thus inducing mitosis during cell cycle progression (17), while PCNA expression has been reported to be a marker of cell proliferation (16). The data of the current study indicated that the expression levels of CDK1 and PCNA were downregulated in RTKN2 knockdown cells, which was consistent with the results of cell proliferation and cell cycle analysis.

Previous studies have demonstrated the anti-apoptotic role of RTKN2 (9-12). Consistent with these observations, the data of the current study demonstrated that knockdown of RTKN2 significantly stimulated the apoptosis of HCC cells (Fig. 4). Invasion is an essential process towards the metastasis of cancer (20). In the current study, reduction of the RTKN2 expression in HCC cells reduced their invasive ability (Fig. 5).

Taken together, the current study indicated that RTKN2 was overexpressed in HCC tissues. In addition, it was demonstrated for the first time, to the best of our knowledge, that RTKN2 served an important role in tumorigenesis and metastasis of HCC. Whether RTKN2 can be used as a diagnostic marker or therapeutic target for HCC remains to be further investigated.

\section{References}

1. Jemal A, Bray F, Center MM, Ferlay J, Ward E and Forman D: Global cancer statistics. CA Cancer J Clin 61: 69-90, 2011.

2. Bosch FX, Ribes J, Díaz M and Cléries R: Primary liver cancer: Worldwide incidence and trends. Gastroenterology 127 (5 Suppl 1): S5-S16, 2004.

3. Finn RS: Development of molecularly targeted therapies in hepatocellular carcinoma: Where do we go now? Clin Cancer Res 16: 390-397, 2010.

4. Collier FM, Gregorio-King CC, Gough TJ, Talbot CD, Walder K and Kirkland MA: Identification and characterization of a lymphocytic Rho-GTPase effector: Rhotekin-2. Biochem Biophys Res Commun 324: 1360-1369, 2004.

5. Reid T, Furuyashiki T, Ishizaki T, Watanabe G, Watanabe N, Fujisawa K, Morii N, Madaule P and Narumiya S: Rhotekin, a new putative target for Rho bearing homology to a serine/threonine kinase, PKN and rhophilin in the rho-binding domain. J Biol Chem 271: 13556-13560, 1996.

6. Karlsson R, Pedersen ED, Wang Z and Brakebusch C: Rho GTPase function in tumorigenesis. Biochim Biophys Acta 1796: 91-98, 2009.

7. Liu CA, Wang MJ, Chi $\mathrm{CW}, \mathrm{Wu} \mathrm{CW}$ and Chen JY: Rho/Rhotekin-mediated NF-kappaB activation confers resistance to apoptosis. Oncogene 23: 8731-8742, 2004.

8. Liu CA, Wang MJ, Chi CW, Wu $\mathrm{CW}$ and Chen JY: Overexpression of rho effector rhotekin confers increased survival in gastric adenocarcinoma. J Biomed Sci 11: 661-670, 2004.

9. Collier FM, Baker AJ, Walder K, Stupka N, Martin SD and Kirkland MA: A Rho-GTPase effector, rhotekin-2 (RTKN2) is associated with BMP8b and IL-16 cytokine expression anti increased sensitivity to apoptosis in lymphocytes. In: Blood amer soc hematology $1900 \mathrm{M}$ street. NW suite 200, Washington, DC 20036 USA, pp679A-pp680A, 2007.

10. Collier FM, Loving A, Baker AJ, McLeod J, Walder K and Kirkland MA: RTKN2 induces NF-KappaB dependent resistance to intrinsic apoptosis in HEK cells and regulates BCL-2 genes in human CD4(+) lymphocytes. J Cell Death 2: 9-23, 2009.

11. Li W, Wu YF, Xu RH, Lu H, Hu C and Qian H: miR-1246 releases RTKN2-dependent resistance to UVB-induced apoptosis in HaCaT cells. Mol Cell Biochem 394: 299-306, 2014.

12. Gregorio-King CC, Gough T, Van Der Meer GJ, Hosking JB, Waugh CM, McLeod JL, Collier FM and Kirkland MA: Mechanisms of resistance to the cytotoxic effects of oxysterols in human leukemic cells. J Steroid Biochem Mol Biol 88: 311-320, 2004.

13. Saha SK, Roy S and Khuda-Bukhsh AR: Ultra-highly diluted plant extracts of Hydrastis canadensis and Marsdenia condurango induce epigenetic modifications and alter gene expression profiles in HeLa cells in vitro. J Integr Med 13: 400-411, 2015.

14. Papavasiliou FN and Schatz DG: Cell-cycle-regulated DNA double-strand breaks in somatic hypermutation of immunoglobulin genes. Nature 408: 216-221, 2000.

15. Ye G, Qin Y, Lu X, Xu X, Xu S, Wu C, Wang X, Wang S and Pan D: The association of renin-angiotensin system genes with the progression of hepatocellular carcinoma. Biochem Biophys Res Commun 459: 18-23, 2015.

16. Kubben FJ, Peeters-Haesevoets A, Engels LG, Baeten CG, Schutte B, Arends JW, Stockbrügger RW and Blijham GH: Proliferating cell nuclear antigen (PCNA): A new marker to study human colonic cell proliferation. Gut 35: 530-535, 1994.

17. Ferrell JE Jr, Wu M, Gerhart JC and Martin GS: Cell cycle tyrosine phosphorylation of $\mathrm{p} 34 \mathrm{cdc} 2$ and a microtubule-associated protein kinase homolog in Xenopus oocytes and eggs. Mol Cell Biol 11: 1965-1971, 1991.

18. Wong CC, Wong CM, Au SL and Ng IO: RhoGTPases and Rho-effectors in hepatocellular carcinoma metastasis: ROCK N'Rho move it. Liver Int 30: 642-656, 2010.

19. Grise F, Bidaud A and Moreau V: Rho GTPases in hepatocellular carcinoma. Biochim Biophys Acta 1795: 137-151, 2009.

20. Leber MF and Efferth T: Molecular principles of cancer invasion and metastasis (review). Int J Oncol 34: 881-895, 2009. 